\title{
Nanoscale materials for information storage
}

\author{
A K Petford-Long*, P Shang**, Y G Wang* and N Owen* \\ *Department of Materials, University of Oxford, Parks Road, Oxford OX1 3PH, UK. \\ **IBM Storage Division, 5600 Cottle Road, San Jose, CA 95193, USA.
}

The novel materials being developed to address the need in information storage systems for smaller bit size and faster memory tend to be very complex and consist of many nanometre thick layers with microstructure that is inhomogeneous on a fine scale. Examples of such materials are spin-valve (SV) and spin tunnel junction (STJ) structures which rely on the giant magnetoresistance (GMR) effect [1] and have applications as magnetoresistive read-heads [2] and as memory elements [3]. In their simplest form, SV and STJ structures consist of a ferromagnetic (FM) layer whose magnetisation direction is fixed by an adjacent antiferromagnetic (AF) pinning layer through exchange coupling, separated by a nonmagnetic spacer layer from a second FM layer (sense layer) whose magnetisation can be rotated in a low external magnetic field. The spacer is a metal in an SV and an insulator in an STJ. A further example is that of an array of magnetic dots or antidots [4], which are being developed for potential use as patterned magnetic media, with each dot (or magnetic region between adjacent antidots) supporting a magnetic bit.

The behaviour of these materials relies on the local magnetic domain structure and magnetisation reversal mechanism, and one of the techniques enabling micromagnetic studies at the sub-micron scale is Lorentz transmission electron microscopy (LTEM) which allows the magnetic domain structure and magnetisation reversal mechanism of a FM material to be investigated dynamically in real-time with a resolution of a few $\mathrm{nm}$. This presentation will highlight some recent research in this field. For example, Figure 1 shows a series of LTEM Foucault mode images of the magnetisation reversal of the sense layer in an STJ element (width $2 \mu \mathrm{m}$ ) of structure: $\mathrm{Ta} / \mathrm{NiFe} / \mathrm{MnFe} / \mathrm{NiFe} / \mathrm{Al}_{2} \mathrm{O}_{3} / \mathrm{NiFe} / \mathrm{Ta}(5 / 6 / 10 / 4 / 2.5 / 7 / 5 \mathrm{~nm})$. The arrows indicate the in-situ applied magnetic field direction and the numbers indicate the field value. After applying a positive field, a $360^{\circ}$ domain wall formed by the combination of two $180^{\circ}$ domain walls (imaged as adjacent bright and dark lines). The wall is pinned at a structural defect at the element surface. The field was then increased to +60 Oe and decreased (centre row of images). The $360^{\circ}$ wall defect acted as a domain nucleation site. If the magnetisation cycle was then repeated but with the positive field increased to $+80 \mathrm{Oe}$, the $360^{\circ}$ wall was removed and reversal of the sense layer magnetisation occurred at a higher negative field. This shows the difficulty of producing large arrays of STJ elements for memory applications, in which the magnetisation reversal field of all the elements must be the same.

Figure 2 shows a Foucault mode LTEM image of an antidot array with $0.1 \mu \mathrm{m}$ width holes. The array was formed using a focused ion beam (FIB) system to mill a series of holes in a thin $\mathrm{Ni}_{80} \mathrm{Fe}_{20}$ film. Competition occurs between the spatially varying demagnetising field arising because of the free poles induced on the anti-dot edges and the intrinsic uniaxial anisotropy. Micromagnetic simulations have predicted remanent states should exist when the demagnetising and anisotropy fields are of the same order. Regular, well defined remanent domains (one is arrowed) can be seen between the anti-dots in the image; these may be suitable for information storage applications.

\section{References}

[1] R L White, IEEE Trans. Mag. 30(2) (1994) 346.

[2] J A Brug, T C Anthony and J H Nickel, MRS Bulletin 21 (1996) 23.

[3] J M Daughton, Thin Solid Films 216 (1992) 162.

[4] R P Cowburn, A Adeyeye and J A C Bland, J. Magn. Magn. Mater. 173 (1997) 193.

[5] This research was supported by the Engineering and Physical Sciences Research Council and Hewlett-Packard Labs, Palo Alto. 


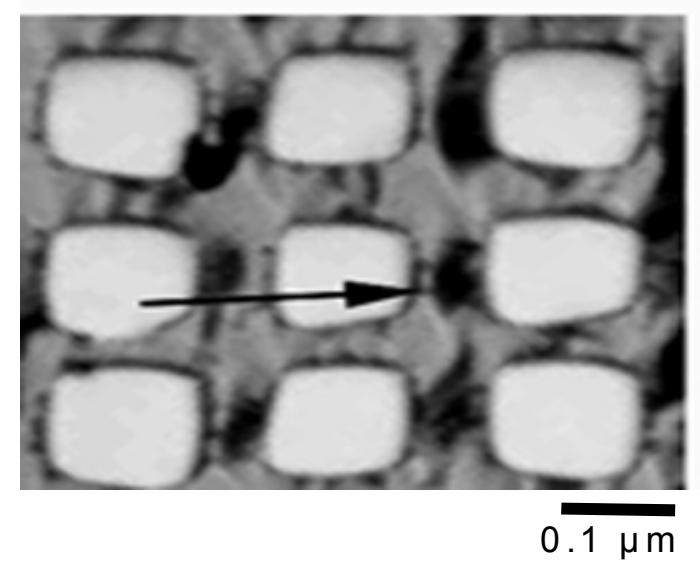

Fig. 1. Foucault mode LTEM images showing in-situ magnetisation reversal of the sense layer in a $\mathrm{MnFe} / \mathrm{NiFe} / \mathrm{Al}_{2} \mathrm{O}_{3} / \mathrm{NiFe}$ STJ element (width $2 \mu \mathrm{m}$ ). Arrows indicate applied field direction.

\begin{tabular}{rrrrr}
+14 & +16 & +18 & +26 \\
-6 & +4 & +7 & +15 & \\
\hline-4 & 0 & +4 &
\end{tabular}

Fig. 2. Foucault mode LTEM image of antidot array cut into a thin Ni80Fe20 film. The magnetic field has been applied along the hard axis of the film. A remanent state domain between two antidots is arrowed. 